\title{
Fração da carga global do diabetes mellitus atribuível ao excesso de peso e à obesidade no Brasil
}

\author{
Andreia Ferreira de Oliveira, ${ }^{1}$ Joaquim Gonçalves Valente ${ }^{1}$ \\ e luri da Costa Leite 1
}

Como citar Oliveira AF, Valente JG, Leite IC. Fração da carga global do diabetes mellitus atribuível ao excesso de peso e à obesidade no Brasil. Rev Panam Salud Publica. 2010;27(5):338-44.

RESUMO Objetivo. Estimar a carga global do diabetes mellitus (DM) para o período de 2002 a 2003 e calcular, para o mesmo período, a fração do diabetes atribuível ao excesso de peso e à obesidade para o Brasil e suas regiões.

Métodos. A prevalência de excesso de peso e obesidade por sexo e faixa etária ( $>20$ anos) e os riscos relativos (obtidos de estudos internacionais) para o desenvolvimento do DM atribuíveis ao excesso de peso e à obesidade foram utilizados para o cálculo da carga global do diabetes. A prevalência de excesso de peso e obesidade para o Brasil e suas regiões foi obtida da Pesquisa de Orçamento Familiar. Foram calculados, para o DM, os anos de vida ajustados para incapacidade (disability-adjusted life years, DALY) a partir da soma de duas parcelas: anos de vida perdidos por morte prematura (years of life lost, $Y L L$ ) e anos de vida perdidos devido à incapacidade (years lived with disability, YLD).

Resultados. Do total de DALY estimados para o DM no Brasil, 70\% provinham dos YLD. Para o Brasil como um todo, 61,8 e 45,4\% do DM no sexo feminino foram atribuíveis a excesso de peso e obesidade, respectivamente. No sexo masculino, esses percentuais foram de 52,8 e $32,7 \%$. As maiores frações atribuíveis foram encontradas nas regiões Sul, Sudeste e CentroOeste e para o grupo populacional entre 35 a 44 anos de idade.

Conclusão. Grande parte da carga do diabetes é atribuível a fatores de risco evitáveis. Medidas voltadas para a prevenção e controle desses fatores de risco, como o excesso de peso e a obesidade, devem estar inseridas na agenda de saúde pública brasileira.

Palavras-chave Sobrepeso; obesidade; anos de vida perdidos por incapacidade; anos potenciais de vida perdidos; diabetes mellitus; Brasil.

O diabetes mellitus (DM) é uma síndrome de etiologia múltipla, decorrente

\footnotetext{
Fundação Oswaldo Cruz, Escola Nacional de Saúde Pública (ENSP/FIOCRUZ), Departamento de Epidemiologia e Métodos Quantitativos. Enviar correspondência para Andréia Ferreira de Oliveira no seguinte endereço: Escola Nacional de Saúde Pública, Departamento de Epidemiologia e Métodos Quantitativos em Saúde, Rua Leopoldo Bulhões 1480/815, CEP 21041-210, Manguinhos, Rio de Janeiro, RJ, Brasil. E-mail: andreiaf@ensp. fiocruz.br, andreiaf@globo.com
}

da falta de insulina ou da incapacidade da insulina de exercer adequadamente os seus efeitos. Suas complicações crônicas decorrem de alterações micro e macrovasculares que levam a disfunção, dano ou falência de vários órgãos $(1,2)$.

Em 2004, estima-se que, no mundo, $44 \%$ das mortes prematuras e $34 \%$ da carga global de doença tenham sido resultado do efeito conjunto de 24 fatores de risco (3). Dentre esses fatores, o hábito de fumar, o colesterol elevado, o excesso de peso, a ingestão elevada de álcool, a hipertensão arterial e a inatividade física são importantes fatores de risco para o desenvolvimento de doenças crônicas, como câncer (endometrial, ovário, mama, cervical, próstata, colorretal, bexiga, pâncreas, hepático, renal), doenças cardiovasculares, doenças respiratórias crônicas e também DM (4-10). A maior parte desses fatores pode ser evitada e 
modificada por meio de mudanças relacionadas ao estilo de vida, visto que estão fortemente relacionados aos padrões de vida e, particularmente, aos de consumo $(5,11)$.

Uma associação positiva entre obesidade e risco de desenvolvimento de DM tipo 2 tem sido recorrentemente observada em estudos transversais (12-15) e prospectivos (15-18). O risco de desenvolver DM cresce continuamente com o aumento do índice de massa corporal (IMC) e da idade (19). Na presença de um IMC acima de $22 \mathrm{~kg} / \mathrm{m}^{2}$, o risco de diabetes aumenta $25 \%$ para cada elevação de $1,0 \mathrm{~kg} / \mathrm{m}^{2}(20,21)$. Além disso, o risco para o desenvolvimento de complicações crônicas está diretamente associado à elevação no IMC. Dos indivíduos acometidos pelo diabetes, 80 a $90 \%$ têm excesso de peso $(6,22,23)$.

Na América Latina e Caribe, no ano de 2001, o DM esteve entre as 10 principais causas de morte (5\% do total de mortes) (24). Nos Estados Unidos, estudos apontam que, entre homens e mulheres, respectivamente, 64 e $74 \%$ dos casos de DM tipo 2 poderiam ser prevenidos por meio de controle do excesso de peso $(25,10)$.

Dada a relevância do DM como problema de saúde pública, e sendo o excesso de peso e a obesidade fatores de risco importantes no seu desenvolvimento, este estudo teve como objetivos estimar a carga do DM para o período de 2002 a 2003 e calcular, para o mesmo período, a fração dessa doença atribuível ao excesso de peso e à obesidade no Brasil e em cada região.

\section{MATERIAIS E MÉTODOS}

A carga global do DM devida ao excesso de peso e à obesidade foi calculada com base na fração populacional atribuível (FPA), que tem por objetivo medir hipoteticamente o quanto da carga de uma doença poderia ser evitado se alguns de seus determinantes pudessem ser eliminados (26). A FPA é definida como a quantidade ou a proporção da incidência ou do risco da doença na população que se pode atribuir a um determinado fator de exposição. No caso em questão, utilizou-se, para seu cálculo, a prevalência do excesso de peso e da obesidade por sexo e faixa etária, assim como os riscos relativos para o desenvolvimento do DM atribuíveis ao excesso de peso e à obesidade.

A FPA foi calculada da seguinte forma:

$$
\mathrm{FPA}=\frac{\sum_{i=0}^{k} p_{i}\left(R R_{i}-1\right)}{1+\sum_{i=0}^{k} p_{i}\left(R R_{i}-1\right)}=1-\frac{1}{\sum_{i=0}^{k} p_{i}\left(R R_{i}\right)}
$$

onde $p_{i}$ é a prevalência da i-ésima categoria de exposição do fator de risco e $R R_{i}$ é o seu respectivo risco relativo em relação à categoria de exposição de referência.

\section{Estimativas de prevalência de excesso de peso e obesidade}

As informações sobre as prevalências do excesso de peso e da obesidade para o Brasil e suas regiões, segundo o sexo e a faixa etária (acima de 20 anos), foram provenientes da Pesquisa de Orçamento Familiar (POF 2002-2003) (27). A POF foi estruturada de modo a propiciar resultados representativos para o Brasil, suas regiões, unidades da federação (total e situação urbana), regiões metropolitanas (nove) e capitais das unidades da federação (situação urbana), incorporando coleta de medidas de peso e estatura para avaliação do estado nutricional dos brasileiros com 20 anos de idade ou mais.

Neste trabalho foram utilizados os pesos e as alturas mensurados na população acima de 20 anos, excluindo gestantes e lactantes. Indivíduos que apresentaram valores de IMC acima de $25,0 \mathrm{~kg} / \mathrm{m}^{2}$ foram classificados na categoria excesso de peso, e aqueles com IMC acima de $30 \mathrm{~kg} / \mathrm{m}^{2}$, como obesos. Com base nas informações da POF, as prevalências do excesso de peso e obesidade foram calculadas para Brasil e suas regiões segundo sexo e faixa etária (28).

\section{Estimativas de risco relativo}

Como não foram encontrados estudos brasileiros com estimativas de risco relativo (RR) para o excesso de peso e a obesidade no desenvolvimento do DM, optou-se por utilizar os resultados obtidos em um estudo realizado nos Estados Unidos por Field et al. (29). Durante 10 anos, esse estudo acompanhou mulheres e homens de meia idade, em uma coorte de enfermeiras e em outra coorte de outros profissionais de saúde, objetivando avaliar os riscos provenientes do excesso de peso. A medida de associação no estudo de Field et al. (29) foi a razão de chances (odds ratio, OR) ajustada para variáveis como idade, fumo e raça.

Entretanto, para o cálculo da fração atribuível, é necessária a medida do RR.
Portanto, as ORs encontradas no estudo de Field et al. (29) foram transformadas em RR, conforme metodologia apresentada por Zhang e Yu (30) e empregada por Sichieri et al. (31) para estimar a carga de hospitalização associada a sobrepeso e obesidade no Brasil.

O estudo realizado por Field et al. (29) não apresenta os RR segundo faixa etária. Por isso, foram adotadas "estruturas" de riscos relativos apresentadas por Yoon et al. (32). No presente estudo, os RR foram apresentados separadamente para sobrepeso e obesidade, tendo sido necessária uma ponderação segundo as prevalências de sobrepeso e obesidade, por faixa etária, obtidas pela POF 20022003, na obtenção dos RR para excesso de peso.

A tabela 1 apresenta as estimativas de RR para o excesso de peso e a obesidade no desenvolvimento do DM, segundo o sexo e a faixa etária. Tomando-se como categoria de referência a faixa etária de 20 a 29 anos, foram obtidos os pesos dos RR nas faixas subsequentes: 30 a 39 anos, 40 a 49 e 50 ou mais, por meio da divisão dos RR apresentados por Yoon et al. (32). Esses pesos resultantes foram aplicados a uma população padrão (calculada com base na média aritmética entre as projeções populacionais do período de 2002 a 2003, disponibilizadas pelo IBGE), obtendo-se assim o total da população exposta ao risco de ocorrência de DM por faixa etária. O risco médio foi obtido dividindo-se esse total pela população de 2002 a 2003. Este risco médio e o peso dos RR foram aplicados ao RR total para a ocorrência de diabetes utilizado no estudo de Schieri et al. (31) (excesso de peso: $\mathrm{RR}=3,7$ para o sexo masculino e 5,1 para o sexo feminino; obesidade: $R R=6,6$ para o sexo masculino e 7,6 para o sexo feminino), por meio de uma regra de três. Com isso, foram obtidos os RR para o desenvolvimento do DM associado ao excesso de peso e à obesidade para cada faixa etária e sexo (RR estimados utilizados). Como o estudo de Schieri et al. (31) trabalha com as categorias de sobrepeso e obesidade, foi necessária uma ponderação dos RR daquele estudo pela prevalência da POF 2002 e 2003 para obtenção do RR total para excesso de peso (IMC > 25).

Conforme a metodologia apresentada por Zhang e Yu (30), também foram calculados os intervalos de confiança de 95\% (IC95\%) da fração atribuível para o excesso de peso e a obesidade, segundo 
TABELA 1. Risco relativo (RR) para desenvolvimento do diabetes mellitus na presença de excesso de peso e obesidade segundo faixa etária e sexo, Brasil, 2002 e 2003

\begin{tabular}{|c|c|c|c|c|c|c|}
\hline \multirow[b]{2}{*}{ Faixa etária (anos) } & \multirow[b]{2}{*}{$\mathrm{RR}^{\mathrm{a}}$} & \multirow{2}{*}{$\begin{array}{l}\text { Peso do } \\
\text { RR }\end{array}$} & \multirow{2}{*}{$\begin{array}{c}\text { Média de } \\
\text { população } \\
\text { 2002-2003 }\end{array}$} & \multirow{2}{*}{$\begin{array}{l}\text { População } \\
\text { expostab }^{\text {b }}\end{array}$} & \multicolumn{2}{|c|}{ RR estimado } \\
\hline & & & & & Masculino & Feminino \\
\hline \multicolumn{7}{|l|}{ Excesso de peso } \\
\hline 20 a 29 & 3,1 & 1,0 & 31089534 & 31089534 & 4,3 & 5,9 \\
\hline 30 a 39 & 2,9 & 0,950 & 26202284 & 24992169 & 4,1 & 5,6 \\
\hline 40 a 49 & 2,5 & 0,808 & 19936973 & 16109074 & 3,5 & 4,8 \\
\hline$\geq 50$ & 2,1 & 0,665 & 27882727 & 18542013 & 2,9 & 3,9 \\
\hline Obesidade & 6,69 & 1,0 & 31089534 & 31089534 & 9,43 & 10,86 \\
\hline 20 a 29 & 4,90 & 0,732 & 26202284 & 19180071 & 6,90 & 7,95 \\
\hline \multicolumn{7}{|l|}{30 a 39} \\
\hline 40 a 49 & 4,09 & 0,611 & 19936973 & 12181490 & 5,76 & 6,63 \\
\hline$\geq 50$ & 2,67 & 0,400 & 27882727 & 11153090 & 3,77 & 4,34 \\
\hline Total & & & 105111517 & 73604185 & & \\
\hline Risco médio & & & & & \multicolumn{2}{|c|}{0,700} \\
\hline
\end{tabular}

a Referência 32.

${ }^{\text {b }}$ População exposta = peso do risco relativo multiplicado pela média da população em 2002 a 2003.

sexo e faixa etária, com base nos limites inferiores e superiores das razões de chances (odds ratios, OR) apresentadas no estudo realizado por Field et al. (29).

Em seguida, as frações atribuíveis brutas foram calculadas (dados não apresentados) e padronizadas para o Brasil e suas regiões, segundo sexo, faixa etária e categoria de IMC (excesso de peso e obesidade), utilizando as prevalências e os riscos relativos já apresentados. As frações foram padronizadas para idade, objetivando comparações entre as regiões do país. Para isso utilizaram-se estimativas de prevalência padronizadas, obtidas através da aplicação à média da população brasileira para o período 2002 a 2003 da prevalência bruta, segundo sexo e faixa etária, conforme a POF 2002-2003.

\section{Estimativa de anos de vida perdidos e anos vividos ajustados por incapacidade}

$\mathrm{O}$ indicador utilizado nos estudos de carga global de doença são os anos de vida ajustados para incapacidade (disability-adjusted life years, DALY). Esse indicador estende o conceito de anos potenciais de vida perdidos por morte prematura (33) ao contemplar anos equivalentes de vida saudáveis perdidos devido a problemas de saúde ou incapacidade. Neste trabalho, o número de DALY foi calculado a partir da soma de duas parcelas: anos de vida perdidos por morte prematura (years of life lost, YLL) e anos de vida perdidos devido à incapacidade (years lived with disability, YLD).
Os dados de mortalidade utilizados para calcular as estimativas de YLL foram obtidos do Banco de Dados do Sistema Único de Saúde (DATASUS) e referem-se aos óbitos ocorridos de 2002 a 2003. O primeiro passo incluiu a redistribuição dos óbitos com idade, sexo e município ignorados e a redistribuição das causas de óbitos por sinais, sintomas e afecções mal definidas.

Três grandes grupos de doenças são comumente utilizados em estudos de carga global: doenças infecciosas e parasitárias, causas maternas, causas perinatais e deficiências nutricionais (grupo I); doenças crônicas não transmissíveis (grupo II); e causas externas (grupo III). Nesta pesquisa, o grupo II foi dividido em DM e demais doenças do grupo II, com base nos critérios estabelecidos no Estudo de Carga Global de Doença (34), utilizando-se como base a Décima Revisão da Classificação Internacional de Doenças (CID-10).

Para calcular o YLL para as regiões do Brasil segundo sexo e faixa etária, a expectativa de vida (utilizada pelo Estudo de Carga Global de Doença) (34) foi multiplicada pela média anual do número de óbitos corrigidos para o período 2002 a 2003. Como não estavam disponíveis as estimativas do componente de morbidade, o YLD, para o período de 2002 a 2003, optamos por estimar as taxas de YLD por 1000 habitantes. Para isso, o número de YLD calculados para o estudo Carga Global de Doença no Brasil (34) foi dividido pelo número de habitantes em 1998, segundo sexo, faixa etá- ria e grandes grupos (GG) de causas de doenças e agravos. Essas taxas foram aplicadas às médias das populações específicas no período 2002 a 2003 para obtenção do número de YLD em cada subpopulação e área geográfica específica.

A taxa de desconto de $3 \%$, proposta na metodologia dos estudos de carga global de doença (33), foi incorporada nos cálculos de mortalidade (YLL) e morbidade (YLD).

\section{RESULTADOS}

No período de 2002 e 2003 ocorreram 2472044 óbitos no Brasil, com uma média anual de 1236022 óbitos, sendo 903806 classificados dentro do grupo II, das doenças crônicas não transmissíveis (DCNT). Esses óbitos deram origem a uma média anual de 12084004 anos de vida perdidos por morte prematura (YLL) e uma média anual de 16262877 anos de vida perdidos por incapacidade (YLD), correspondendo a uma taxa de DALY de 161,3 para cada 1000 habitantes. A tabela 2 descreve a carga global das doenças dos grupos I, II e III no período estudado.

A tabela 3 apresenta o número de DALY entre os indivíduos acima de 20 anos segundo os grupos de doenças. Observa-se que, do total de DALY encontrados nas regiões brasileiras, a maior parte está associada ao grupo II. Nas regiões Norte, Sudeste e Sul, a porcentagem de DALY para diabetes em relação ao total de DALY do grupo II foi superior a $10 \%$ (tabela 3 ). 
TABELA 2. Carga global para grupos de doenças, Brasil, 2002 a $2003^{a}$

\begin{tabular}{|c|c|c|c|c|c|}
\hline Grupo $^{b}$ & Óbitos & $Y^{\prime} L^{c}$ & YLD $^{C}$ & DALYc & $\begin{array}{c}\text { DALYc/1 } 000 \\
\text { habitantes }\end{array}$ \\
\hline Grupo I & 205650 & 4413716 & 4170763 & 8584479 & 48,8 \\
\hline Grupo II - Diabetes & 58670 & 722561 & 1619252 & 2341813 & 13,3 \\
\hline Grupo II - Demais & 845137 & 11361443 & 14643625 & 26005068 & 148,0 \\
\hline Grupo III & 126567 & 2997935 & 1140628 & 4138563 & 23,5 \\
\hline Total & 1236022 & 19495655 & 21574268 & 41069923 & 233,7 \\
\hline
\end{tabular}

TABELA 3. Número absoluto e percentuais de anos de vida ajustados para incapacidade em indivíduos acima de 20 anos segundo região do Brasil e grupos de doenças, 2002 a 2003

\begin{tabular}{|c|c|c|c|c|c|c|c|c|}
\hline \multirow[b]{3}{*}{ Região } & \multirow{3}{*}{$\begin{array}{c}\text { DALYa } \\
\text { total }\end{array}$} & \multirow{3}{*}{$\begin{array}{l}\text { DALYa } \\
\text { Grupo I }\end{array}$} & \multirow{2}{*}{\multicolumn{2}{|c|}{$\begin{array}{c}\text { DALYa } \\
\text { Grupo II }\end{array}$}} & \multirow{3}{*}{$\begin{array}{c}\text { DALYa } \\
\text { Grupo III }\end{array}$} & \multicolumn{3}{|c|}{ Diabetes } \\
\hline & & & & & & \multirow[b]{2}{*}{ DALYa } & \multirow{2}{*}{$\begin{array}{c}\% \text { DALYa/ } \\
\text { total }^{\mathrm{d}}\end{array}$} & \multirow{2}{*}{$\begin{array}{l}\text { \% DALYa/ } \\
\text { Grupo II }\end{array}$} \\
\hline & & & No. & $\%^{c}$ & & & & \\
\hline Norte & 1821404 & 288985 & 1361455 & 74,7 & 170963 & 138278 & 7,6 & 10,2 \\
\hline Nordeste & 8720965 & 1166178 & 6901315 & 79,1 & 653473 & 607993 & 7,0 & 8,8 \\
\hline Sudeste & 13840180 & 1608029 & 10839491 & 78,3 & 1392661 & 1105566 & 8,0 & 10,2 \\
\hline Sul & 4226402 & 473839 & 3345373 & 79,2 & 407190 & 358043 & 8,5 & 10,7 \\
\hline Centro-Oeste & 1824373 & 231376 & 1369332 & 75,1 & 223665 & 113277 & 6,2 & 8,3 \\
\hline Brasil & 30433324 & 3768407 & 23816966 & 78,3 & 2847951 & 2323157 & 7,6 & 9,8 \\
\hline
\end{tabular}

a DALY: anos de vida perdidos ajustados para incapacidade.

b Grupo I: doenças infecciosas e parasitárias, causas maternas, causas perinatais e deficiências nutricionais; Grupo II: doenças crônicas não-transmissíveis; Grupo III: causas externas.

c Porcentagem de DALY do grupo II em relação ao DALY total.

d Porcentagem de DALY do diabetes mellitus em relação ao DALY total.

e Porcentagem de DALY do diabetes mellitus em relação ao DALY do grupo II.

TABELA 4. Frações atribuíveis padronizadas (\%) de excesso de peso e obesidade associados ao diabetes para o Brasil e suas regiões segundo sexo, 2002 a 2003

\begin{tabular}{|c|c|c|c|c|}
\hline \multirow[b]{2}{*}{ Região } & \multicolumn{2}{|c|}{ Excesso de peso } & \multicolumn{2}{|c|}{ Obesidade } \\
\hline & Masculino & Feminino & Masculino & Feminino \\
\hline Norte & 50,5 & 59,9 & 30,2 & 42,3 \\
\hline Nordeste & 47,9 & 61,2 & 27,1 & 43,2 \\
\hline Sudeste & 54,6 & 62,0 & 34,9 & 46,6 \\
\hline Sul & 55,1 & 63,3 & 35,6 & 48,0 \\
\hline Centro-Oeste & 53,9 & 60,8 & 32,0 & 41,2 \\
\hline Brasil & 52,8 & 61,8 & 32,7 & 45,4 \\
\hline
\end{tabular}

A tabela 4 apresenta as frações atribuíveis padronizadas para excesso de peso e obesidade associados ao DM para as regiões segundo o sexo. Tanto para o Brasil quanto para suas regiões, o sexo feminino apresentou as maiores frações atribuíveis para excesso de peso e obesidade associados ao diabetes. No Brasil, no sexo feminino, 61 , 8 e $45,4 \%$ do $\mathrm{DM}$, respectivamente, foram atribuíveis a excesso de peso e obesidade. No sexo masculino, esses percentuais foram de 52,8 e $32,7 \%$.

A tabela 5 apresenta as frações atribuíveis de excesso de peso e obesidade as- sociados ao DM para o Brasil e suas regiões, desagregadas segundo sexo e faixa etária. Para o Brasil como um todo, observa-se que as maiores frações atribuíveis para excesso de peso e obesidade, em ambos os sexos, são encontradas nas faixas etárias de 35 a 44 anos. A partir dessa idade, os valores tendem a apresentar queda. Frações atribuíveis superiores à média nacional foram encontrados em quase todas as faixas etárias nas regiões Sudeste e Sul, independentemente do sexo, para excesso de peso e obesidade. Um padrão semelhante foi observado na Região Centro-Oeste apenas para o sexo masculino.

\section{DISCUSSÃO}

Neste artigo, observou-se um peso importante das DCNT no total de DALY estimados para o Brasil e suas regiões. Um resultado semelhante foi encontrado em um estudo realizado na Sérvia no ano de 2000. Jankovic et al. (35) observaram que as doenças isquêmicas do coração e cerebrovasculares, câncer de pulmão, depressão e diabetes foram responsáveis por aproximadamente $70 \%$ da carga global de doença. Entre as mulheres, o diabetes esteve entre as cinco principais doenças responsáveis pela perda de anos de vida por morte prematura e incapacidade.

Stevens et al. (36), no México, constataram que as DCNT causaram $75 \%$ do total das mortes e $68 \%$ do total de DALY. Entre os principais fatores de risco para a carga global de doença estava o IMC elevado, que foi o fator que mais contribuiu para o total de carga de doença $(5,1 \%$ do total de DALY em ambos os sexos, sendo $6,0 \%$ entre as mulheres). Com relação ao 
TABELA 5. Frações atribuiveis do excesso de peso e obesidade associadas ao diabetes para o Brasil e suas regiões segundo sexo e faixa etária, 2002 e 2003

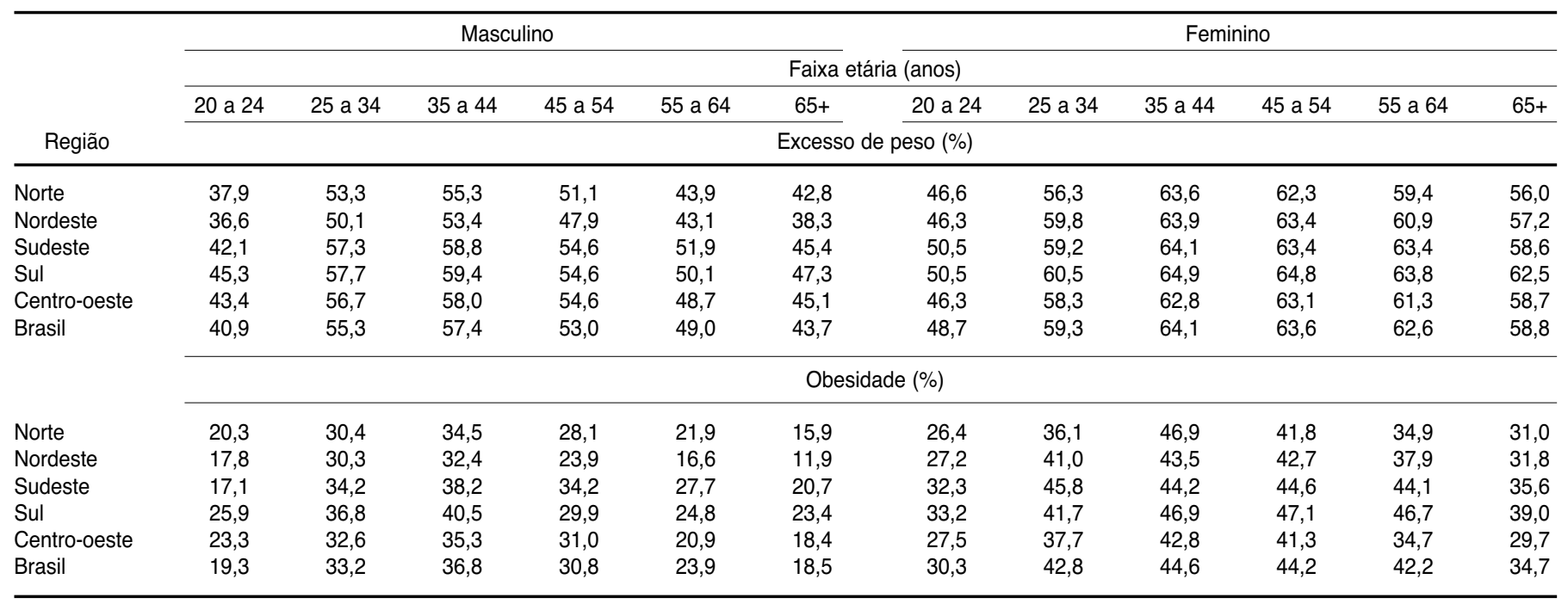

total de DALY, o diabetes foi responsável por 3,5\% do total, sendo $4,2 \%$ entre as mulheres.

Estimativas recentes mostraram que, em todo o mundo, aproximadamente metade da carga de doença é atribuível às DCNT, ou seja do grupo II. O diabetes representou $1,3 \%$ do total de DALY para 2004, estimando-se que aumente para 2,3\% em 2030. Na região das Américas, o diabetes representou $2,9 \%$ do total de DALY (37). Na Nova Zelândia e na Austrália, o diabetes representou de 3,8 a $5,5 \%$ do total de DALY $(38,39)$.

Neste estudo, verificou-se que a participação do grupo II no total dos DALY para 2002 a 2003 é semelhante, ou até mesmo superior, ao encontrado nesses países, chegando a $80 \%$ em algumas regiões. A participação do diabetes no total de DALY no Brasil (7,6\%) também foi superior àquela encontrada nesses países. Na Região Sul do país a participação do diabetes chegou a $8,5 \%$. A transição epidemiológica pode ser uma explicação para esses achados, dada a crescente participação das DCNT no perfil de morbimortalidade das populações em países em desenvolvimento, como é o caso do Brasil.

Com relação ao risco atribuível para o excesso de peso relacionado ao DM, no Canadá, em 1970, 22\% dos casos de diabetes tipo 2 foram atribuíveis à obesidade, sendo que, em 2004, esse percentual subiu para 39\% (40). Também no Canadá, Birmingham et al. (41) observaram que $50,7 \%$ de todos os casos de dia- betes tipo 2 foram atribuídos à obesidade. Na Suíça, 42,5\% dos casos de diabetes foram atribuíveis à obesidade $\left(\mathrm{IMC}>30 \mathrm{~kg} / \mathrm{m}^{2}\right)$ (42).

Nos Estados Unidos, 21\% dos casos de diabetes entre homens foram atribuídos ao excesso de peso, contra apenas 3\% para as mulheres (43). Dados da Organização Mundial da Saúde (OMS) apontam que, nos Estados Unidos, aproximadamente $64 \%$ do DM tipo $2 \mathrm{em}$ homens e $74 \%$ em mulheres poderiam ser evitados caso o IMC fosse mantido abaixo de $25 \mathrm{~kg} / \mathrm{m}^{2}$ (44).

As porcentagens de DM atribuíveis ao excesso de peso neste estudo foram superiores às relatadas na literatura internacional: 61,8 e $52,8 \%$ no sexo feminino e masculino, respectivamente, para o Brasil como um todo. Quanto à fração atribuível à obesidade, os percentuais foram de $45,4 \%$, no sexo feminino e $32,7 \%$ no sexo masculino. Em ambos os sexos, as maiores frações atribuíveis foram observadas nas regiões Sudeste e Sul do país.

Estudos evidenciam que, nos Estados Unidos, o IMC está diretamente relacionado ao risco de DM na vida em ambos os sexos. Naquele país, tanto o sobrepeso quanto a obesidade, particularmente em idades jovens, aumentam o risco na vida de um diagnóstico de diabetes, havendo uma diminuição desse risco com o avanço da idade $(45,46)$.

Neste artigo, observou-se que, para o Brasil e suas regiões, as frações atribuíveis ao excesso de peso e à obesidade aumentaram, em ambos os sexos, até os
44 anos de idade, mantendo-se constantes ou mesmo declinando a partir dessa idade. A exceção foi a Região Sudeste, onde a fração atribuível à obesidade na faixa etária de 35 a 44 anos apresentou ligeira queda. Tal fato já foi discutido por Schieri et al. (31). Esses autores indicam que, por ser a obesidade um problema relativamente recente de saúde pública no Brasil, os grupos populacionais mais velhos ainda não sofreram as consequências crônicas da obesidade ou, talvez, com o envelhecimento, o excesso de peso tenha tendido a ser relativamente menos importante. Dados recentes da OMS (3) mostram que, em países de renda média/alta, incluindo o Brasil, o sobrepeso e a obesidade estão entre os cinco principais fatores de risco com impacto importante na carga global de doença (DALY), além de serem responsáveis pelo aumento no desenvolvimento de doenças crônicas não transmissíveis, como o DM.

As limitações relacionadas à complexidade das interpretações e cálculos da fração atribuível já foram abordadas por alguns pesquisadores $(47,48)$. As estimativas de RR variam de estudo para estudo, dependendo das características da população e das categorias de referência e exposição escolhidas. Portanto, a utilização de RR provenientes de outras populações, que não a brasileira, pode ser considerada uma limitação desta pesquisa. Por outro lado, o RR é um instrumento de grande utilidade em saúde pública por facilitar o estabelecimento de 
prioridades de atuação. Acreditamos que o fato de terem sido usados dados coreanos para realizar cálculos para a população brasileira não tenha gerado resultados imprecisos, já que, possivelmente, a relação "dose-dependente" entre o IMC e o desenvolvimento do DM em grupos de idade mais jovens, em comparação a grupos com idade mais avançada, é semelhante nos dois países (32).

Acredita-se, ainda, que a população brasileira seja parecida com a população dos Estados Unidos (em termos genéticos e de hábitos de vida), justificando-se a projeção da "força de associação", diferenciada por faixa etária do estudo coreano, para os riscos relativos totais ponderados para excesso de peso e obesidade apresentados por Schieri et al. (31). Embora haja variações no RR de uma população para outra, o maior determinante da variação na carga atribuível devido a um fator de risco em particular não é a diferença no RR, mas sim diferenças na distribuição dos níveis da exposição na população (49).
Outra limitação refere-se à utilização, neste trabalho, de estimativas de RR baseadas em um estudo com indivíduos de meia idade. Como os riscos relativos de morte associados a excesso de peso e obesidade são menores em indivíduos mais velhos (50), as estimativas das frações atribuíveis associadas ao diabetes podem estar enviesadas na faixa etária acima de 60 anos. Uma terceira limitação é a incorporação das estimativas de YLD do estudo de carga global de doença realizado no Brasil em 1998 no cálculo da parcela de morbidade referente aos anos de 2002 a 2003. No entanto, acredita-se que essa decisão não tenha modificado em grande escala os dados encontrados, em função do curto período considerado. Finalmente, destaca-se que uma grande variedade de tipos de câncer pode ser atribuída a excesso de peso e obesidade. Porém, na presente investigação foram selecionados, dentro da metodologia de carga global de doença, apenas os códigos de doenças e agravos relacionados ao diabetes e suas complicações.

\section{REFERÊNCIAS}

1. Sociedade Brasileira de Diabetes. Consenso Brasileiro sobre Diabetes 2002: diagnóstico e classificação do diabetes melito e tratamento do diabetes melito tipo 2. Rio de Janeiro: Diagraphic; 2003. Disponível em: diabetes.org.br/ educacao/docs/Consenso_atual_2002.pdf. Acessado em abril de 2010.

2. Engelgau MM, Geiss LS, Saaddine JB, Boyle JP, Benjamin SM, Gregg EW, et al. The evolving diabetes burden in the Unites States. Ann Intern Med. 2004;140(11):945-50.

3. World Health Organization. Global health risks: mortality and burden of disease attributable to selected major risks. Genebra: WHO; 2009.

4. Yusuf S, Reddy S, Ounpuu S, Anand S. Global burden of cardiovascular diseases: part I: general considerations, the epidemiologic transition, risk factors, and impact of urbanization. Circulation. 2001;104(22):2746-53.

5. World Health Organization. The World Health Report 2002: Reducing risks, promoting healthy life. Genebra: WHO; 2002.

6. Organização Pan-Americana da Saúde. Doenças crônico-degenerativas e obesidade: estratégia mundial sobre alimentação saudável, atividade física e saúde. Brasília: OPAS; 2003.

7. Yach D, Hawkes C, Gould CL, Hofman KJ. The global burden of chronic diseases: overcoming impediments to prevention and control. JAMA. 2004;291(21):2616-22.

8. Boutayeb A, Boutayeb S. The burden of non communicable diseases in developing countries. Int J Equity Health. 2005;4(1):2.

9. Yach D, Stuckler D, Brownell KD. Epidemiologic and economic consequences of the global epidemics of obesity and diabetes. Nat Med. 2006;12(1):62-6.

10. World Health Organization. Obesity: preventing and managing the global epidemic. WHO Technical Report Series 894. Genebra: WHO; 2000.

11. Beaglehole R, Yach D. Globalisation and the prevention and control of non-communicable disease: the neglected chronic diseases of adults. Lancet. 2003;362(9387):903-8. Risk factors for the development of type II diabetes among men enrolled in the usual care group of the Multiple Risk Factor Intervention Trial. Diabetes Care. 1993;16(10):1331-9.

13. Chou P, Liao MJ, Tsai ST. Associated risk factors of diabetes in Kin-Hu, Kinmen. Diabetes Res Clin Pract. 1994;26(3):229-35.

14. Collins VR, Dowse GK, Toelupe PM, Imo TT, Aloaina FL, Spark RA, et al. Increasing prevalence of NIDDM in the Pacific Island population of Western Samoa over a 13-year period. Diabetes Care. 1994;17(4):288-96.

15. Canoy B, Buchan I. Challenges in obesity epidemiology. Obes Rev. 2007;8(Suppl 1):1-11.

16. Knowler WC, Pettitt DJ, Saad MF, Charles MA, Nelson RG, Howard BV, et al. Obesity in the Pima Indians: its magnitude and relationship with diabetes. Am J Clin Nutr. 1991;53 (6 Suppl):1543S-51S.

17. Cassano PA, Rosner B, Vokonas PS, Weiss ST. Obesity and body fat distribution in relation to the incidence of non-insulin-dependent diabetes mellitus. A prospective cohort study of men in the normative aging study. Am J Epidemiol. 1992;136(12):1474-86.
12. Shaten BJ, Smith GD, Kuller LH, Neaton JD.
Dada a prevalência crescente do DM e o impacto de suas complicações crônicas em todo o país, é importante um investimento do poder público, incluindo a melhoria do acesso de todas as classes sociais a informações confiáveis sobre os determinantes e consequências do excesso de peso e o desenvolvimento de ações públicas consistentes nos ambientes físicos, econômicos e socioculturais que permitam escolhas saudáveis com relação à dieta e à prática diária de atividades físicas.

As regiões Sul e Sudeste, com as maiores frações atribuíveis ao excesso de peso e obesidade, devem ser foco de intervenções específicas, voltadas principalmente para a prevenção do diabetes e a promoção de hábitos saudáveis de vida. Além disso, as faixas etárias mais precoces devem ser alvo prioritário, visto que os maiores riscos de desenvolvimento do diabetes relacionados a excesso de peso e obesidade ocorrem nesses grupos etários.
18. Chan JM, Rimm EB, Colditz GA, Stampfer MJ, Willett WC. Obesity, fat distribution, and weight gain as risk factors for clinical diabetes in men. Diabetes Care. 1994;17(9): 961-9.

19. Thompson D, Edelsberg J, Colditz GA, Bird $\mathrm{AP}$, Oster G. Lifetime health and economic consequences of obesity. Arch Intern Med. 1999;159(18):2177-83.

20. World Health Organization. Integrated prevention of noncommunicable diseases: draft global strategy on diet, physical activity and health. Executive Board EB113/44 Add.1; 2003. Disponível em: idf.org/integrated-preventionnon-communicable-diseases. Acessado em abril 2010.

21. Finer N. Medical consequences of obesity. Medicine. 2006;34(12):510-4

22. Consenso Latino-Americano em Obesidade. Primeira convenção latino-americana para consenso em obesidade. Rio de Janeiro: Ministério da Saúde; 1998.

23. Khaodhiar L, McCowen KC, Blackburn GL. Obesity and its comorbid conditions. Clin Cornerstone. 1999;2(3):17-31.

24. Lopez AD, Mathers CD, Ezzati M, Jamison DT, Murray CJL. Global burden of disease and risk factors. Washington: Oxford and World Bank; 2006.

25. Visscher TL, Seidell JC. The public health impact of obesity. Annu Rev Public Health. 2001; 22:355-75.

26. Llorca J, Delgado-Rodríguez M. A new way to estimate the contribution of a risk factor in populations avoided nonadditivity. J Clin Epidemiol. 2004;57(5):479-83. 
27. Instituto Brasileiro de Geografia e Estatística. Pesquisa de orçamentos familiares 2002-2003. Microdados. $3^{\mathrm{a}}$ ed. Rio de Janeiro: IBGE; 2007.

28. Oliveira AF. Carga global de doença no Brasil: o papel de fatores de risco como o tabagismo e o excesso de peso [tese]. Rio de Janeiro: Escola Nacional de Saúde Pública; 2009.

29. Field AE, Coakley EH, Must A, Spadano JL, Laird N, Dietz W, et al. Impact of overweight on the risk of developing common chronic diseases during a 10-year period. Arch Intern Med. 2001;161(13):1581-6.

30. Zhang J, Yu KF. What's the relative risk? A method for correcting the odds ratio in cohort studies of common outcomes. JAMA. 1998; 280(19):1690-1.

31. Sichieri R, do Nascimento S, Coutinho W. The burden of hospitalization due to overweight and obesity in Brazil. Cad Saude Publica. 2007;23(7):1721-7.

32. Yoon YS, Shin SA, Noh JH, Oh SW. Burden of type 2 diabetes in young Korean adults owing to obesity. Diabetes Care. 2005;28(9):2329.

33. Murray CJL, Lopez AD. Global comparative assessments in the health sector. Genebra: WHO; 1994.

34. Fundação Instituto Oswaldo Cruz. Projeto carga global de doença no Brasil: relatório técnico final. Rio de Janeiro: ENSP/FIOCRUZ/ FENSPTEC; 2002.

35. Jankovic S, Vlajinac $H$, Bjegovic $V$, Marinkovic J, Sipetic-Grujicic S, MarkovicDenic L, et al. The burden of disease and injury in Serbia. Eur J Public Health. 2006; 17(1):80-5.

36. Stevens G, Dias RH, Thomas KJ, Rivera JA, Carvalho N, Barquera S, et al. Characterizing the epidemiological transition in Mexico: national and subnational burden of diseases, injuries, and risk factors. PloS Med. 2008;5(6): e125.

37. World Health Organization. The global burden of disease: 2004 update. Genebra: WHO; 2008.

38. New Zealand, Ministry of Health. Our health, our future Hauora Pakari, Koiora Roa: the health of New Zealanders 1999. Wellington: New Zealand; 1999.

39. Begg S, Vos T, Barker B, Stevenson C, Stanley $\mathrm{L}$, Lopez AD. The burden of disease and injury in Australia 2003. PHE 82. Canberra: AIHW; 2007.

40. Luo W, Morrison H, de Groh M, Waters C, Desmeules M, Jones-Mclean E, et al. The burden of adult obesity in Canada. Chronic Dis Can. 2007;27(4):135-44.

41. Birmingham CL, Muller JL, Palepu A, Spinelli JJ, Anis AH. The cost of obesity in Canada. CMAJ. 1999;160(4):483-8.

42. Schmid A, Schneider H, Golay A, Keller U. Economic burden of obesity and its comorbidities in Switzerland. Soz Praventivmed. 2005;50(2):87-94.

43. Wilson PW, D'Agostinho RB, Sullivan L, Parise H, Kannel WB. Overweight and obesity as determinants of cardiovascular risk: the
Framingham experience. Arch Intern Med. 2002;162(9):1867-72.

44. World Health Organization. Obesity: preventing and managing the global epidemic. Report of a WHO consultation on obesity. Genebra: WHO; 1998.

45. Narayan KM, Boyle JP, Thompson TJ, Gregg EW, Williamson DF. Effect of BMI on lifetime risk for diabetes in the U.S. Diabetes Care. 2007;30(6):1562-6.

46. Must A, Spadano J, Coakely EH, Field AE, Colditz G, Dietz WH. The disease burden associated with overweight and obesity. JAMA. 1999;282(16):1523-9.

47. Flegal KM. Estimating the impact of obesity. Soz Praventivmed. 2005;50(2):73-4.

48. Levine B. What does the population attributable fraction mean? Prev Chronic Dis. 2007; 4(1):A14.

49. Murray CJ, Lopez AD. Global health statistics: a compendium of incidence, prevalence and mortality estimates for over 200 conditions. Genebra: WHO; 1996.

50. Bender R, Jockel KH, Trautner C, Spraul M, Berger M. Effect of age on excess mortality in obesity. JAMA. 1999;281(16):1498-504.

Manuscrito recebido em 17 de agosto de 2009. Aceito em versão revisada em 28 de dezembro de 2009.
ABSTRACT

\section{Fraction of the global burden of diabetes mellitus attributable to overweight and obesity in Brazil}

Key words
Objective. To estimate the global burden of diabetes mellitus (DM) in 2002-2003 and to calculate the fraction of diabetes attributable to excess weight and obesity for Brazil and its regions.

Methods. The prevalence of excess weight and obesity according to sex and age (> 20 years) and the relative risks for the development of DM (derived from international studies) attributable to excess weight and obesity were used to calculate the global burden of DM. The prevalence of excess weight and obesity for Brazil and its regions was obtained from the Family Budget Survey (Pesquisa de Orçamento Familiar). Disability-adjusted life years (DALY) were calculated for DM based on the sum of two components: years of life lost (YLL) and years lived with disability (YLD).

Results. Of the total DALY estimated for DM in Brazil, YLD accounted for $70 \%$. For Brazil as a whole, $61.8 \%$ and $45.4 \%$ of DM in females was attributable to excess weight and obesity, respectively. Among males, the proportions were $52.8 \%$ and $32.7 \%$. The largest excess weight/obesity-attributable fractions were observed in the South, Southeast, and Midwest regions and in the 35-44-year-old age groups.

Conclusions. A large fraction of the burden of DM is attributable to preventable individual risk factors. Measures targeting prevention and control of risk factors such as excess weight and obesity must be included in the Brazilian public health agenda.

Overweight; obesity; disability-adjusted life years; potential years of life lost; diabetes mellitus; Brazil. 